\title{
Learning difficulties: The effect on mental health of children
}

\author{
Apergi Marioleni \\ Graduate Department of the University of Patras, Athens, Greece
}

Email address:

marioleniapergi@yahoo.gr

\section{To cite this article:}

Apergi Marioleni. Learning Difficulties: The Effect on Mental Health of Children. American Journal of Nursing Science. Special Issue: Mental Health Care: Aspects, Challenges and Perspectives. Vol. 4, No. 2-1, 2015, pp. 12-15. doi: 10.11648/j.ajns.s.2015040201.13

\begin{abstract}
The following article is an approach to the subject of learning difficulties and its influence on the mental health of children. Through this article the characteristics of children with learning difficulties are highlighted and problems that are created in their mental health. It approximates the factors of perception, memory and language, to which children experience most such issues. It highlights the problems that arise in the mental health of children and their impact on the social environment. Finally, findings are presented and recommendations are given for addressing this phenomenon, like the reliable diagnosis and psychological support of each individual.
\end{abstract}

Keywords: Learning Difficulties, Characteristics, Mental Health

\section{Introduction}

The term learning difficulties was used the first time from Samuel Kirk to 1962 (in the project Education Exceptional Children) so as to identify "a delay or impaired development in one or more functions of the written or spoken words or mathematics, because of a possible cerebral dysfunction or behavior disturbances and feelings".[1]

Later other definitions were developed of researchers mainly in the U.S.A. and it is widely accepted that "learning difficulties is a general term to a heterogeneous group of disorders that manifest as difficulties in learning and use of speech, reading, writing, reasoning or mathematical abilities. Regarding the extent of the problem findings suggest that about $20-25 \%$ of the population appears to have a problem of difficulty in a field or object learning .[2]

The characteristics of people with learning difficulties are many and at times there have been efforts to group them . However not all these people develop the same symptoms and may not be easy to develop them. However most researchers distinguish some features which are likely to occur with greater frequency and that can simultaneously create mental health problems in children. Such characteristics are : low self esteem, poor emotional control, the intense stress reactions without much thought, poor adaptation to new situations, poor relationships with peers, etc.[3]

\section{Characteristics of Children with Learning Difficulties}

a) Disturbances in one or more basic psychological processes such as Perceptual-mnemonic functions, language processing, etc.

b) Learning disorders are not due to sensory impairment, mental retardation, and emotional disturbances.

c) Mismatch in the development of individual skills and expected performance.

d) Normal intelligence.

e) Hyperactivity.

f) Disorders of attention and concentration.

g) Perceptual difficulties with coordination of general weakness.

h) Specific difficulties in school subject.

i) Emotional instability.

j) Disturbances of memory and thinking.[4]

Perception and Memory: The cognitive functions of perception and memory are inextricably linked with the learning of any kind and therefore their involvement seems very likely. In many cases learning difficulties and in particular those referred to in reading, spelling and mathematics. In recent years research has focused on treatment processes of visual and auditory stimuli. 


\subsection{Visual Perception}

The main areas of visual perception in which problems occur are : the relationship of space perception, visual discrimination, visual memory and visual sequence. So those who have problems understanding relationships in space have difficulties to perceive objects in the room, to distinguish the right and the left, the direction, distance and speed . Regarding to the visual distinction it refers to the ability to distinguish objects based on some characteristics. In visual memory, difficulties arise in storage and recall of information visual engaged. Difficulties in understanding sequences of objects, symbols or facts presented or represented visually are observed to the impaired visual sequence.[5]

\subsection{Auditory Perception}

People with learning difficulties and in particular reading disabilities may face challenges in auditory perception. Most research conducted examines the headphones deficits as opposed to visual and auditory memory problems reported and audio sequences.[3].

Language: The language is particularly relevant to the issue of learning difficulties. It is estimated that about $50 \%$ of people with learning difficulties have language problems or language deficits, which may be referred to one or more aspects of language, such as phonology, morphology, semantics, syntax or in the function of language as a communication medium.[3].

\subsection{In the Phonological Level}

Presents difficulties in articulation is often observed.

\subsection{In Shape Level}

The person may have problems in using suffixes numbers and names, adverbs, verbs of years.

\subsection{Drafting Not}

The use of simpler and poorer proposals .[6]

\subsection{At the Semantic Level}

Difficulties occur with the use of linguistics expressions, which include mainly simple and affirmative formalities.

Memory: Memory is the ability one has to encode, process and recall information that has been exposed at some time . The memory model under the polydomiko, consists of three parts: the short-term, long-term and working memory, among which occur on three discrete processes which are the encoding ,the storage and the recall.[7,8]

According to the model polydomiko, memory information arriving from the environment to sensory registers (ear, eye, etc.), which is available for a minimum period. With the help of attention, some of them are strengthened while others are forgotten. The strengthened information are led to the first part of the memory system the short term memory. In such a limited amount of information (6-9 parts) are stored for a limited time (usually less than 20 seconds).

Then after the committing a limbo elements of information that are not reinforced and the stimuli-information are encoded and stored in semantic (cognitive) patterns in longterm memory. In this memory is longer becomes the permanent storage of data information takes place. The new information is incorporated to the existing knowledge by completing and updating it or by removing the one that was false.[3]

The difficulties faced by people with learning difficulties in mnemonic capacity are related (in combination with these phonological awareness) with reading and spelling and language problems. The mnemonic difficulties extend the entire mnemonic mechanism.[9,10,11]

In the short term memory people with learning difficulties have poor performance in projects that require linguistic processing and especially when the interval between the stimulus presentation and recall is high. Such long memory problems arise from the inefficient use of phonological codes along with the limited capacity of short-term memory and poor use of internal strategies of repetition and organization .[7]

In the working memory, there are major problems in the mnemonic device of people with learning difficulties. They experience problems with the sequence of phonemes withdrawal, letters, words and actual pseudo-words related to reading. Even the problems in the implementation process of withdrawal are associated with the tracking of the general mnemonic process. Many scientists believe that the problems are based on the inability of coding the information and the reduced incentives, placing such people in a demanding mental effort.[3]

Problems of attention and concentration: The attention and concentration problems are so intense that people with learning difficulties can be put in the same group with the ones having Attention Deficit Disorder with or without Hyperactivity.

\section{Problems in the Mental Health of Children}

Most researchers distinguish some features which may occur with greater frequency in the personality and behavior of children with learning disabilities. Children and adults with learning disabilities are not exempt from experiencing mental health problems. In fact, children with learning disabilities are at much greater risk of having mental health problems than the general population. Children and young people with learning disabilities are also much more likely to live in poverty, to have few friends and to have additional long term health problems and disabilities such as epilepsy and sensory impairments. Children with learning disabilities can find it hard to build social relationships, and are more likely to say that they have difficulties getting on with their peers than children without learning disabilities. A learning disability is also likely to reduce a child's capacity for 
finding creative and adaptive solutions to life's challenges. All of these factors are known to have a negative impact on mental health, putting people with learning disabilities at greater risk of developing mental health problems. [12].Some of these are:

Self-esteem: People with learning difficulties feel that several times they are trying to cope with everyday proficiency, requirements, but more often believe that they are inferior to the circumstances. Our society values certain accomplishments, such as achieving high social status, independence, employment, relationships and a family. People with learning disabilities may have difficulty achieving these things, which may affect their self esteem.

Social acceptance: Factors that slow the development of positive social relationships are many among which them being the inability to express feelings, low self esteem etc. The low social acceptance has been attributed to various reasons. Among those highlighted are the difficulties these people have in learning and knowledge acquisition, any negative behavior, lack of skills of specific types of behavior.[13,14]

The problematic behavior: It features about $50 \%$ of cases and is influenced by the type and nature of the problems. There are lesions EGO child as phobias, distress, depression and aggression which are created due to the impact of negative experiences during the learning process.

Physical health problems transitory illness/infections: Physical disabilities and illness are increased in people with learning disabilities. They may cause long-term pain or discomfort to the individual - for example, people with Down's syndrome are prone to chest infections. The effects of physical impairments are exaggerated by a lack of understanding in wider society, such as through poor accessibility to buildings and facilities.[15]

Medication: People with learning disabilities are likely to receive medication for a variety of physical, neurological and psychiatric reasons. The side effects of medication, particularly when the person is in receipt of two or more psychotropic medications, need to be considered as they can contribute to mental health problems.[15]

Self-image: People with learning disabilities may feel they are different to other people. Some may have physical disabilities that set them apart from others, or may feel that they are inferior because they are more reliant on the support of others. A poor self-image can be a catalyst for a mental health problem. $[3,15]$

\section{Addressing}

Addressing the learning disabilities is perhaps the most important aspect of the whole matter and the ultimate goal of all our efforts. However, successful treatment of learning disabilities is not always easy, because it is dependent on the cause, characteristics and intensity of difficulties. Parents and teachers should be very careful in how those characteristics on performance and behavior occur and should be put in alert «vigilance» to proceed to more detailed scrutiny of performance evaluation. Particular attention is required in those cases where the performance of a person in one area is not consistent with the amount of effort made by him and his family. The degree of alertness in such cases should be even higher.

The measures we must take to lead to a more effective assessment of learning difficulties are the following:

1. Reliable diagnosis by people who are trained in the field of learning disabilities is a necessity for reliable characterization of individuals with reading, spelling and other learning difficulties.

2. Psychological support for anyone experiencing learning difficulties, is the principle that should govern the implementation of any intervention program for the treatment of learning disabilities. This principle is generic and refers to any learning disability regardless of its peculiarities. Indeed given that many children with learning difficulties may have a negative perception of themselves and their abilities, then the psychological support of the child by both the teacher and the parents should be taken for granted. Mistakes and failures of the child should be treated as normal phenomena of their situation and as inevitable elements of their effort to learn.[3]

3. The mental health of children with learning difficulties should be preserved and is the foundation of any systematic effort to assist these individuals. The activities provided should be relevant and of practical value for people with learning difficulties, and such that will help in future to communicate better with other people and socialize. It is therefore necessary to create an environment that fosters the belief in the child that mistakes and failures are not only accepted, but expected, and should be seen as opportunities for learning. In other words to pursue the difficult task to indeed convince the children that are vulnerable to failures-many of whom feel defeated and tired after years of disappointments and failures- that failures that can lead to success.

4. Early foresight: Early identification of people with learning disabilities is considered the most important factors that has a positive impact and improves the quality of life of these people as well as anticipating the emergence of secondary problems, which affect the primary difficulty.[16]

5. Regular updates of teachers and parents on issues related to learning, disorders and the children' $\mathrm{s}$ temperament combined with systematic cooperation with professional staff. Thus some mild learning difficulties in the initial symptoms can be quickly detected and lead people to the pupils' failures. For this reason both teachers and parents need to be aware of the types of learning disabilities, their characteristics and the reasons that cause them, so as to be sure of the diagnosis and effective in their interventions.[16] Parents and teachers should be aware of all the issues and define the disorder: developmental, learning 
behavior and emotional. We also observed the child in all events of his life, in order to have an as complete as possible picture of their abilities and weaknesses.

\section{Conclusions}

Learning disabilities is a pronounced phenomenon usually interpreted wrong or underestimated resulting in many difficulties for children and impacts on their mental health. . It is therefore necessary to take measures of dealing with this phenomenon in order to reduce problems in the lives of children and acceptance their by the social context. All people with learning disabilities have the same rights to learning, education and knowledge acquisition.

\section{References}

[1] S.A. Kirk , Education exceptional children. Boston : Houghton Mifflin, 1962

[2] J. H Meier , Prevalence and characteristics of learning disabilities found in second grade children. "Journal of Learning Disabilities", 1971 4; 4 pp. 1-16.

[3] Porpodas, Learning and Knowledge in Education, cognitive analysis-difficulties-applications, 2011, pp.559, 560,564-566, 569-570.

[4] E., Kakoula M.Ed., .Psychoeducational treatment of learning disabilities, the reports of the Greek institu of applied pedagogy and education «Learning how to learn», May 2010.

[5] P. Satz, .\&R. Morris, Learning Disabilities subtypes : A review. In F.J. Pirozzolo \& M.C. Wittrock (Eds.), Neuropsycological and cognitive processes in reading, New York : Academic Press, 1981, pp.109-144
[6] E.H Wiig, , \& E.M Semel, , Language assessment and intervention for the learning disabled ( 2 nd ed.) Boston : Allyn \& Bacon, 1984

[7] H.L. Swanson, J.B. Cooney \& J.K, McNamara , Learning disabilities and memory. B.Y. L. Wong(ed.) Learning about learning disabilities (3 ${ }^{\text {rd }}$ ed.), San Diego, CA: Elsevier, 2004, pp.41-92

[8] D. Sousa, How the special needs brain learns. Thousand Oaks, CA :Corw in Press, 2001

[9] H.L. Swanson, "Journal of Learning Disabilities" ,Short-term memory and working memory. Do both contribute to our understanding of academic achievement in children and adults with learning disabilities? 1994, pp. 27, 34-50.

[10] A.D. Baddeley, Working memory. London, UK: Oxford University Press, 1986

[11] S.Ceci, M. Ringstorm \& S. Lea "Journal of Learning Disabilities" Do language learning disabled children have impaired memories? In search of underlying process., 1981, pp.14, 159-163.

[12] Emerson and Hatton, The mental health of children and adolescents with Learning Disabilities in Britain. Institute for health Reasearch, Lancaster University, 2007.

[13] W.N. Bender, Daily grading in mainstream classes. The Directive Teacher, 6 (2), 1984, pp. 4-5

[14] G.M. Morrison, S.R. Forness, \& D.L, MacMillam, "Journal of Education Psychology", Influences on the sociometric rating of mildly handicapped children : A path analysis. 1983, pp.75, 63-74.

[15] Mental health nursing of adults with learning disabilities, Royal college of nursing, 2010.

[16] E. Livaniou. Learning dificulties and behavioral issues in classroom settings. Kedros. Athens 2004. 